Claremont Colleges

Scholarship@ Claremont

All HMC Faculty Publications and Research

HMC Faculty Scholarship

$12-1-2005$

\title{
Recounting the Odds of an Even Derangement
}

Arthur T. Benjamin

Harvey Mudd College

Curtis D. Bennet

Loyola Marymount University

Florence Newberger

California State University - Long Beach

\section{Recommended Citation}

Benjamin, Arthur T., Curtis D. Bennet and Florence Newberger. "Recounting the Odds of an Even Derangement." Mathematics Magazine, Vol 78, No. 5, pp. 387-390, December 2005.

This Article is brought to you for free and open access by the HMC Faculty Scholarship at Scholarship @ Claremont. It has been accepted for inclusion in All HMC Faculty Publications and Research by an authorized administrator of Scholarship @ Claremont. For more information, please contact scholarship@cuc.claremont.edu. 
Recounting the Odds of an Even Derangement

Author(s): Arthur T. Benjamin, Curtis T. Bennett and Florence Newberger

Source: Mathematics Magazine, Vol. 78, No. 5 (Dec., 2005), pp. 387-390

Published by: Mathematical Association of America

Stable URL: http://www.jstor.org/stable/30044197

Accessed: 11/06/2013 18:35

Your use of the JSTOR archive indicates your acceptance of the Terms \& Conditions of Use, available at http://www.jstor.org/page/info/about/policies/terms.jsp

JSTOR is a not-for-profit service that helps scholars, researchers, and students discover, use, and build upon a wide range of content in a trusted digital archive. We use information technology and tools to increase productivity and facilitate new forms of scholarship. For more information about JSTOR, please contact support@ jstor.org. 
We see the potential value of this proof as twofold. First, it appears cleaner and shorter than what is found in most texts. And our constant, $\ln (8)$, is modest compared to Sierpinski's 4 [7], Apostol's 6 [1], or the $32 \ln (2)$ offered in earlier editions of Niven and Zuckerman [6]. LeVeque [5], Hardy and Wright [4], and the latest edition of Niven and Zuckerman [6] give no particular constant, merely proving that one exists. Chebychev [3] achieved a much smaller constant than ours, but with considerably more effort. We hope that our short proof will be found to have pedagogical value.

\title{
REFERENCES
}

1. Tom M. Apostol, Introduction to Analytic Number Theory, Springer-Verlag, New York, 1976.

2. Bela Bollobas, Erdős first paper: A proof of Bertrand's Postulate, pre-print, 1998.

3. P.L. Chebychev, Memoire sur les nombres premiers, J. Math. Pures et Appl. 17, (1852), 366-390.

4. G.H. Hardy and E.M. Wright, An Introduction to the Theory of Numbers, 5th Edition, Clarendon Press, Oxford, 1979.

5. William J. LeVeque, Fundamentals of Number Theory, Dover Publications, New York, 1977.

6. I. Niven and H.S. Zuckerman, An Introduction to the Theory of Numbers, 3rd, 4th, 5th Editions, Wiley, New York, 1972, 1980, 1991.

7. Waclaw Sierpinski, Elementary Theory of Numbers, PWN, Warsaw, 1964.

\section{Recounting the Odds of an Even Derangement}

\author{
ARTHUR T. BENJAMIN \\ Harvey Mudd College \\ Claremont, CA 91711 \\ benjamin@hmc.edu
}

\section{CURTIS T. BENNETT}

Loyola Marymount University Los Angeles, CA 90045 cbennett@Imu.edu

\author{
FLORENCE NEWBERGER \\ California State University \\ Long Beach, CA 90840-1001 \\ fnewberg@csulb.edu
}

Odd as it may sound, when $n$ exams are randomly returned to $n$ students, the probability that no student receives his or her own exam is almost exactly $1 / e$ (approximately 0.368 ), for all $n \geq 4$. We call a permutation with no fixed points, a derangement, and we let $D(n)$ denote the number of derangements of $n$ elements. For $n \geq 1$, it can be shown that $D(n)=\sum_{k=0}^{n}(-1)^{k} n ! / k !$, and hence the odds that a random permutation of $n$ elements has no fixed points is $D(n) / n$ !, which is within $1 /(n+1)$ ! of $1 / e$ [1].

Permutations come in two varieties: even and odd. A permutation is even if it can be achieved by making an even number of swaps; otherwise it is odd. Thus, one might even be interested to know that if we let $E(n)$ and $O(n)$ respectively denote the number of even and odd derangements of $n$ elements, then (oddly enough),

$$
E(n)=\frac{D(n)+(n-1)(-1)^{n-1}}{2}
$$


and

$$
O(n)=\frac{D(n)-(n-1)(-1)^{n-1}}{2} .
$$

The above formulas are an immediate consequence of the equation $E(n)+O(n)=$ $D(n)$, which is obvious, and the following theorem, which is the focus of this note.

THEOREM. For $n \geq 1$,

$$
E(n)-O(n)=(-1)^{n-1}(n-1) .
$$

Proof 1: Determining a Determinant The fastest way to derive equation (1), as is done in [3], is to compute a determinant. Recall that an $n$-by- $n$ matrix $A=\left[a_{i j}\right]_{i, j=1}^{n}$ has determinant

$$
\operatorname{det}(A)=\sum_{\pi \in S_{n}} a_{1 \pi(1)} a_{2 \pi(2)} \cdots a_{n \pi(n)} \operatorname{sgn}(\pi),
$$

where $S_{n}$ is the set of all permutations of $\{1, \ldots, n\}, \operatorname{sgn}(\pi)=1$ when $\pi$ is even, and $\operatorname{sgn}(\pi)=-1$ when $\pi$ is odd. Let $A_{n}$ denote the $n$-by- $n$ matrix whose nondiagonal entries are $a_{i j}=1$ (for $i \neq j$ ), with zeroes on the diagonal. For example, when $n=4$,

$$
A_{4}=J_{4}-I_{4}=\left(\begin{array}{llll}
1 & 1 & 1 & 1 \\
1 & 1 & 1 & 1 \\
1 & 1 & 1 & 1 \\
1 & 1 & 1 & 1
\end{array}\right)-\left(\begin{array}{llll}
1 & 0 & 0 & 0 \\
0 & 1 & 0 & 0 \\
0 & 0 & 1 & 0 \\
0 & 0 & 0 & 1
\end{array}\right)=\left(\begin{array}{llll}
0 & 1 & 1 & 1 \\
1 & 0 & 1 & 1 \\
1 & 1 & 0 & 1 \\
1 & 1 & 1 & 0
\end{array}\right)
$$

By (2), every permutation that is not a derangement will contribute 0 to the sum (since it uses at least one of the diagonal entries), every even derangement will contribute 1 to the sum, and every odd derangement will contribute -1 to the sum. Consequently, $\operatorname{det}\left(A_{n}\right)=E(n)-O(n)$. To see that $\operatorname{det}\left(A_{n}\right)=(-1)^{n-1}(n-1)$, observe that $A_{n}=J_{n}-I_{n}$, where $J_{n}$ is the matrix of all ones and $I_{n}$ is the identity matrix. Since $J_{n}$ has rank one, zero is an eigenvalue of $J_{n}$, with multiplicity $n-1$, and its other eigenvalue is $n$ (with an eigenvector of all 1s). Apply $J_{n}-I_{n}$ to the eigenvectors of $J_{n}$ to find the eigenvalues of $A_{n}:-1$ with multiplicity $n-1$ and $n-1$ with multiplicity 1 . Multiplying the eigenvalues gives us $\operatorname{det}\left(A_{n}\right)=(-1)^{n-1}(n-1)$, as desired.

A 1996 Note in the MAGAZINE [2] gave even odder ways to determine the determinant of $A_{n}$.

Although the proof by determinants is quick, the form of (1) suggests that there should also exist an almost one-to-one correspondence between the set of even derangements and the set of odd derangements.

Proof 2: Involving an Involution Let $D_{n}$ denote the set of derangements of $\{1, \ldots, n\}$, and let $X_{n}$ be a set of $n-1$ exceptional derangements (that we specify later), each with sign $(-1)^{n-1}$. We exhibit a sign reversing involution on $D_{n}-X_{n}$. That is, letting $T_{n}=D_{n}-X_{n}$, we find an invertible function $f: T_{n} \rightarrow T_{n}$ such that for $\pi$ in $T_{n}, \pi$ and $f(\pi)$ have opposite signs, and $f(f(\pi))=\pi$. In other words, except for the $n-1$ exceptional derangements, every even derangement "holds hands" with an odd derangement, and vice versa. From this, it immediately follows that $\left|E_{n}\right|-\left|O_{n}\right|=(-1)^{n-1}(n-1)$.

Before describing $f$, we establish some notation. We express each $\pi$ in $D_{n}$ as the product of $k$ disjoint cycles $C_{1}, \ldots, C_{k}$ with respective lengths $m_{1}, \ldots, m_{k}$ for some 
$k \geq 1$. We follow the convention that each cycle begins with its smallest element, and the cycles are listed from left to right in increasing order of the first element. In particular, $C_{1}=\left(\begin{array}{llll}1 & a_{2} & \cdots & a_{m_{1}}\end{array}\right)$ and, if $k \geq 2, C_{2}$ begins with the smallest element that does not appear in $C_{1}$. Since $\pi$ is a derangement on $n$ elements, we must have $m_{i} \geq 2$ for all $i$, and $\sum_{i=1}^{k} m_{i}=n$. Finally, since a cycle of length $m$ has sign $(-1)^{m-1}$, it follows that $\pi$ has sign $(-1)^{\sum_{i=1}^{k}\left(m_{i}-1\right)}=(-1)^{n-k}$.

Let $\pi$ be a derangement in $D_{n}$ with first cycle $C_{1}=\left(\begin{array}{llll}1 & a_{2} & \cdots & a_{m}\end{array}\right)$ for some $m \geq 2$. We say that $\pi$ has extraction point $e \geq 2$ if $e$ is the smallest number in the set $\{2, \ldots, n\}-\left\{a_{2}\right\}$ for which $C_{1}$ does not end with the numbers of $\{2, \ldots, e\}-\left\{a_{2}\right\}$ written in decreasing order. Note that $\pi$ will have extraction point $e=2$ unless the number 2 appears as the second term or last term of $C_{1}$. We illustrate this definition with some pairs of examples from $D_{9}$. Notice that in each pair below, the number of cycles of $\pi$ and $\pi^{\prime}$ differ by one, and the extraction point $e$ occurs in the first cycle of $\pi$ and is the leading element of the second cycle of $\pi^{\prime}$.

$$
\begin{aligned}
& \pi=(19728)(36)(45) \text { and } \pi^{\prime}=(197)(28)(36)(45) \text { have } e=2 \text {. } \\
& \pi=(1297385)(46) \text { and } \pi^{\prime}=(1297)(385)(46) \text { have } e=3 \text {. } \\
& \pi=(1973852)(46) \text { and } \pi^{\prime}=(1972)(385)(46) \quad \text { have } e=3 \text {. } \\
& \pi=(1948532)(67) \text { and } \pi^{\prime}=(1932)(485)(67) \quad \text { have } e=4 \text {. } \\
& \pi=(1495832)(67) \text { and } \pi^{\prime}=(14932)(58)(67) \quad \text { have } e=5 \text {. } \\
& \pi=(138697542) \quad \text { and } \pi^{\prime}=(138542)(697) \quad \text { have } e=6 \text {. }
\end{aligned}
$$

Observe that every derangement $\pi$ in $D_{n}$ contains an extraction point unless $\pi$ consists of a single cycle of the form $\pi=\left(\begin{array}{lll}1 & a_{2} Z\end{array}\right)$, where $Z$ is the ordered set $\{2,3, \ldots, n-1, n\}-\left\{a_{2}\right\}$, written in decreasing order. For example, the 9-element derangement (1 59876432 ) has no extraction point. Since $a_{2}$ can be any element of $\{2, \ldots, n\}$, there are exactly $n-1$ derangements of this type, all of which have sign $(-1)^{n-1}$. We let $X_{n}$ denote the set of derangements of this form. Our problem reduces to finding a sign reversing involution $f$ over $T_{n}=D_{n}-X_{n}$.

Suppose $\pi$ in $T_{n}$ has extraction point $e$. Then the first cycle $C_{1}$ of $\pi$ ends with the (possibly empty) ordered subset $Z$ consisting of the elements of $\{2, \ldots, e-1\}-\left\{a_{2}\right\}$ written in decreasing order. Our sign reversing involution $f: T_{n} \rightarrow T_{n}$ can then be succinctly described as follows:

$$
\left(1 a_{2} X e Y Z\right) \sigma \stackrel{f}{\longleftrightarrow}\left(1 a_{2} X Z\right)(e Y) \sigma
$$

where $X$ and $Y$ are ordered subsets, $Y$ is nonempty, and $\sigma$ is the rest of the derangement $\pi$.

Notice that since the number of cycles of $\pi$ and $f(\pi)$ differ by one, they must be of opposite signs. The derangements on the left side of (3) are those for which the extraction point $e$ is in the first cycle. In this case, $Y$ must be nonempty, since otherwise " $e Z$ " would be a longer decreasing sequence and $e$ would not be the extraction point. The derangements on the right side of (3) are those for which the extraction point $e$ is not in the first cycle (and must therefore be the leading element of the second cycle). In this case, $Y$ is nonempty since $\pi$ is a derangement. Thus for any derangement $\pi$, the derangement $f(\pi)$ is also written in standard form, with the same extraction point $e$ and with the same associated ordered subset $Z$. Another way to see that $\pi$ and $f(\pi)$ have opposite signs is to notice that $f(\pi)=(x y) \pi$ (multiplying from left to right), where $x$ is the last element of $X\left(x=a_{2}\right.$ when $X$ is empty), and $y$ is the last element 
of $Y$. Either way, $f(f(\pi))=\pi$, and $f$ is a well-defined, sign-reversing involution, as desired.

In summary, we have shown combinatorially that for all values of $n$, there are almost as many even derangements as odd derangements of $n$ elements. Or to put it another way, when randomly choosing a derangement with at least five elements, the odds of having an even derangement are nearly even.

Acknowledgment. We are indebted to Don Rawlings for bringing this problem to our attention and we thank Magnhild Lien, Will Murray, and the referees for many helpful ideas.

\title{
REFERENCES
}

1. R. A. Brualdi, Introductory Combinatorics, 3rd ed., Prentice-Hall, New Jersey, 1999.

2. C. A. McCarthy and A. T. Benjamin, Determinants of the tournaments, this MAGAZINE, 69 (1996), 133-135.

$\rightarrow$ C. D. Olds, Odd and even derangements, Solution E907, Amer. Math. Monthly, 57 (1950), 687-688.

\section{Volumes of Generalized Unit Balls}

\author{
XIANFU WANG \\ UBC Okanagan \\ 3333 University Way, Kelowna \\ B.C., Canada, V1V 1 V7 \\ shawn.wang@ubc.ca
}

Diamonds, cylinders, squares, stars, and balls. These geometric figures are familiar to undergraduate students, but what could they possibly have in common? One answer is: They are generalized balls. The standard Euclidean ball can be distorted into a variety of strange-shaped balls by linear and nonlinear transformations. The purpose of this note is to give a unified formula for computing the volumes of generalized unit balls in $n$-dimensional spaces.

A generalized unit ball in $\mathbb{R}^{n}$ is described by the set

$$
\mathbb{B}_{p_{1} p_{2} \ldots p_{n}}=\left\{\mathbf{x}=\left(x_{1}, \ldots, x_{n}\right):\left|x_{1}\right|^{p_{1}}+\cdots+\left|x_{n}\right|^{p_{n}} \leq 1\right\}
$$

where $p_{1}>0, p_{2}>0, \ldots, p_{n}>0$.

When the numbers $p_{1}, \ldots, p_{n}$ are all greater than or equal to 1 , the unit ball $\mathbb{B}_{p_{1} \ldots p_{n}}$ is convex. Since $|x|^{p}$ is not concave on $[-1,1]$ for $0<p<1, \mathbb{B}_{p_{1} \ldots p_{n}}$ is not necessarily convex anymore when $n>1$. When $p_{1}=p_{2}=\cdots=p_{n}=p \geq 1$, we obtain the usual $l_{p}$ ball. The $l_{2}$ ball is denoted by $\mathbb{B}$. By choosing different numbers $p_{i}$, we can alter the appearance of the generalized balls greatly, as shown in FIGURE 1 with examples in $\mathbb{R}^{3}$.

Motivated by an article by Folland [5], I derived a unified formula for calculating the volume of these balls. Although the volume formulas for the standard Euclidean ball $\mathbb{B}$ and simplex have been known for a long time [4, pp. 208, 220], the unified formula is (relatively) new. It is surprising that no matter how strange the balls look, the volume of any ball can be computed by a single formula, as follows:

THEOREM. Assume $p_{1}, \ldots, p_{n}>0$. The volume of the unit ball $\mathbb{B}_{p_{1} p_{2} \ldots p_{n}}$ in $\mathbb{R}^{n}$ is equal to 